\title{
A Search for Tidal Stellar Debris from the Magellanic Clouds: Survey Results from the First Two Years
}

\author{
S. R. Majewski ${ }^{1}$, J. C. Ostheimer \\ University of Virginia, Department of Astronomy, Charlottesville, VA \\ 22903-0818, USA
}

W. E. Kunkel

Las Campanas Observatory, Carnegie Observatories, La Serena, Chile

K. V. Johnston

Institute for Advanced Study, Princeton, NJ 08540, USA

R. J. Patterson and C. Palma

University of Virginia, Department of Astronomy, Charlottesville, VA 22903-0818, USA

\section{Introduction}

An important discriminant between leading models for the origin of the Magellanic Stream is the presence of a stellar counterpart to the HI gas stream: ram pressure stripping of gas by a putative hot Galactic halo would act only on Magellanic gas while gravitational tidal stripping would act on both gas and stars. Several previous attempts to find tidal stellar debris have failed to find carbon stars, A stars, or other main sequence stars in the Magellanic Stream (Mathewson et al. 1979; Recillas-Cruz 1982; Brück \& Hawkins 1983; Guhathakurta \& Lin 1999). However, there has long been a suggestion (Kunkel 1979; LyndenBell 1982) of a possible Magellanic association of satellite galaxies and globular clusters that have similar orbits and may derive from the break up of a greater Magellanic galaxy (Lynden-Bell \& Lynden-Bell 1995; Majewski et al. 1997). Recent models (Moore \& Davis 1994; Johnston 1998) of the tidal disruption of Large Magellanic Cloud (LMC)-like systems indicate a wide dispersal of debris, much wider than the rather confined HI stream, so that the contrast of tidal debris against the Galactic fore/background would be low. If true, this could explain some of the previous negative results for tidal debris searches.

\section{Survey and Results to Date}

Results of these recent dynamical models drive the strategy of our new attempt to find stellar tidal debris from the Magellanic Clouds. To detect widely dis-

\footnotetext{
${ }^{1}$ Visiting Associate, Carnegie Observatories; Packard Foundation Fellow; Cottrell Scholar
} 


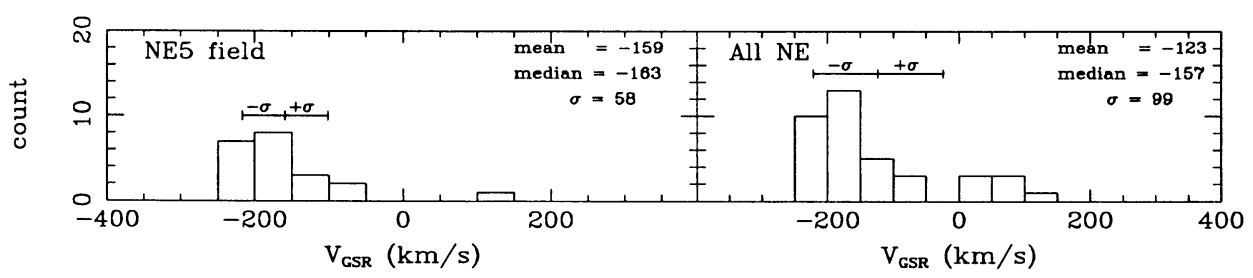

Figure 1. The radial velocity distribution of giants in a single field (left panel) northeast of the LMC $\left(l=271^{\circ}, b=-19^{\circ}\right)$, and for a total of five fields in the same region of the sky (right panel)

persed, low contrast debris requires that large areas be surveyed. Presently, one may cover large areas practically only with small telescopes, but to search for debris at the distance of the Magellanic Clouds then requires bright stars as targets. We have therefore begun a photometric search for giant stars in a $\sim 25^{\circ}$ radius ring of fields around the Clouds. We have used the Las Campanas 1-m Swope telescope to image in the DDO51 and Washington broadband filters, a combination that effectively separates $\mathrm{K}$ and $\mathrm{M}$ giants from dwarfs, since the DDO51 filter is centered on the surface gravity sensitive $\mathrm{Mg} b$ triplet and $\mathrm{MgH}$ stellar features around $5100 \AA$. To date we have imaged $\sim 25 \mathrm{deg}^{2}$ in 29 fields to Washington $M \sim 19.5$. Giant star candidates are observed with the Modular Spectrograph on the DuPont 2.5-m telescope, from which we obtain $10-15 \mathrm{~km}$ $\mathrm{s}^{-1}$ radial velocities and checks on the surface gravity.

Figure 1 shows the radial velocity distribution (corrected for Local Standard of Rest) of giants in a single field northeast of the LMC $\left(l=271^{\circ}, b=-19^{\circ}\right)$, and for a total of five fields in the same region of the sky. The distributions clearly do not follow expectations for a random halo field population - i.e., a Gaussian with $\sim 110 \mathrm{~km} \mathrm{~s}^{-1}$ dispersion centered on 0 ; rather, they seem to show dominance by $a$ negative velocity "moving group". Moreover, the distance-velocity distributions we obtain for these and our other fields roughly match the expected distributions for multiply-wrapped, tidal arms as predicted by our semi-analytical LMC debris model (see Johnston 1998). However, while we conclude from these first survey results that we are finding correlated streams of halo stellar debris around the Magellanic Clouds, much more work is needed before we may associate these streams definitively with the Clouds. But the apparent lack of an appreciable random field star population in our survey data at least suggests that tidal debris makes a predominant contribution to the outer parts of the Milky Way.

\section{References}

Brück, M. T., \& Hawkins, M.R.S. 1983, A\&A, 124, 216

Guhathakurta, P., \& Lin, D. N. C. 1999, in preparation

Johnston, K. V. 1998 ApJ, 495, 297

Kunkel, W. E. 1979, ApJ, 228, 718 
Lynden-Bell, D. 1982, Observatory, 102, 202

Lynden-Bell, D., \& Lynden-Bell, R.M. 1995, MNRAS, 275, 429

Majewski, S. R., Phelps, R.L., \& Rich, R.M. 1997, ASP Conf. Ser., 112, 1

Mathewson, D.S., et al. 1979, in IAU Symp. 84, 547 (ed. W. B. Burton)

Moore, B., \& Davis, M. 1994, MNRAS, 270, 209

Recillas-Cruz, E. 1982, A\&A, 124, 216 